\title{
EXISTENCE OF POSITIVE EVANESCENT SOLUTIONS TO SOME QUASILINEAR ELLIPTIC EQUATIONS
}

\author{
OCTAVIAN G. MUSTAFA
}

(Received 15 October 2007)

\section{Abstract}

We establish that the elliptic equation

$$
\Delta u+f(x, u)+g(|x|) x \cdot \nabla u=0
$$

defined in an exterior domain of $\mathbb{R}^{n}, n \geq 3$, has a positive solution which decays to 0 as $|x| \rightarrow+\infty$ under quite general assumptions upon $f$ and $g$.

2000 Mathematics subject classification: 34A12, 35J60.

Keywords and phrases: positive solution, nonlinear elliptic equation, exterior domain.

\section{Introduction}

Let us consider the quasilinear elliptic differential equation

$$
\Delta u+f(x, u)+g(|x|) x \cdot \nabla u=0, \quad x \in G_{A},
$$

where $G_{A}=\left\{x \in \mathbb{R}^{n}:|x|>A\right\}, A>0$ and $n \geq 3$. The existence of positive solutions of equation (1), either bounded or decaying to 0 (a phenomenon called evanescence), has been investigated by several authors; see [1, 2, 4, 5, 9].

It has been established (see $[1,2]$ ) that it is sufficient for the functions $f, g$ to be Hölder continuous or continuously differentiable in order to analyze the asymptotic behavior of the solutions to equation (1) by the comparison method [7]. To set the general hypotheses, we assume that there exist the continuous functions $a$ : $[A,+\infty) \rightarrow[0,+\infty)$ and $w:[0,+\infty) \rightarrow[0,+\infty)$ such that

$$
0 \leq f(x, u) \leq a(|x|) w(u), \quad x \in G_{A}, u \in[0, \zeta],
$$

for a certain $\zeta>0$. We assume that $w$ is monotone nondecreasing, $w(0)=0$ and $w(u)>0$ for any $u>0$.

The author's research was financed by Romanian AT grant 97GR/25.05.2007 with CNCSIS code 100 .

(C) 2008 Australian Mathematical Society 0004-9727/08 \$A2.00+0.00 
In $[1$, p. 150$]$, A. Constantin asked also that $w \in C^{1}([0,+\infty),[0,+\infty))$,

$$
\int_{1}^{+\infty}\left(\frac{1}{w(s)}\right) d s=+\infty
$$

and $g$ be bounded. Then, in the circumstances given by the condition

$$
\int_{A}^{+\infty} s[a(s)+|g(s)|] d s<+\infty,
$$

the existence of a positive solution to equation (1) was proved. In a further work [2, p. 335], it was established that this positive solution actually decays to 0 as $|x| \rightarrow+\infty$. By making use of the elementary inequality $w(u) \leq \sup \left\{\left|w^{\prime}(s)\right|: s \in\right.$ $[0, \zeta]\} \cdot u$ for all $u \in[0, \zeta]$, the integral condition regarding $w$ was successfully removed.

Another improvement was obtained by M. Ehrnström [4, Lemma 3.2] who concluded that, still for $w \in C^{1}$, the boundedness restriction upon $g$ can be avoided. Moreover, if $g$ takes only nonnegative values, hypothesis (3) reduces to

$$
\int_{A}^{+\infty} s a(s) d s<+\infty
$$

see [4, Theorem 3.3].

It is important to remark at this point that by asking $w$ to be merely continuous we could cover cases such as that where $w(u)=u^{\lambda}$ for any $u \in[0,1]$ and $\lambda \in(0,1)$. A result for simply continuous $w$ 's was obtained in [5, Theorem 1]; however, its main hypothesis is much more restrictive than (3) unless certain restrictions are imposed upon $g$, for example $\lim _{r \rightarrow+\infty} r g(r)>0$; see [5, Remark 2].

Our aim in this note is to demonstrate a variant of Constantin's and Ehrnström's results in the case where $w$ is only continuous and $g$ is nonnegative-valued.

\section{A special ordinary differential equation}

In the spirit of $[1,4,9]$, the heart of our proof relies upon a result concerning the positive solutions to the ordinary differential equation

$$
v^{\prime \prime}+b(t) w\left(\frac{v}{t}\right)=0, \quad t \geq t_{0} \geq 1 .
$$

Proposition 1. Assume that the function $b:\left[t_{0},+\infty\right) \rightarrow[0,+\infty)$ is continuous and such that

$$
\int_{t_{0}}^{+\infty} b(t) d t<+\infty
$$


Then, given $c>0$, equation (5) has a solution $v(t)$ defined in $\left[t_{0},+\infty\right)$ that verifies the inequalities

$$
v(t) \geq c, \quad v^{\prime}(t) \leq \frac{v(t)-c}{t} \quad \text { for all } t \geq t_{0},
$$

and is developable as

$$
v(t)=o(t) \quad \text { when } t \rightarrow+\infty .
$$

PROOF. Suppose that $t_{0} \geq 1$ is large enough so that

$$
\int_{t_{0}}^{+\infty} b(\tau) d \tau \leq \frac{c}{w(2 c)}
$$

We introduce the set

$$
D=\left\{v \in C\left(\left[t_{0},+\infty\right), \mathbb{R}\right) \mid c \leq v(t) \leq 2 c t \text { for every } t \geq t_{0}\right\} .
$$

A partial order on $D$ is given by the usual pointwise order ' $\leq$ ', that is, we say that $v_{1} \leq v_{2}$ if and only if $v_{1}(t) \leq v_{2}(t)$ for all $t \geq t_{0}$, where $v_{1}, v_{2} \in D$. It is not hard to see that $(D, \leq)$ is a complete lattice.

For the operator $V: D \rightarrow C\left(\left[t_{0},+\infty\right), \mathbb{R}\right)$ with the formula

$$
V(v)(t)=c+t \int_{t}^{+\infty} \frac{1}{s^{2}} \int_{t_{0}}^{s} \tau b(\tau) w\left(\frac{v(\tau)}{\tau}\right) d \tau d s, \quad v \in D, t \geq t_{0},
$$

we may write

$$
\begin{aligned}
c & \leq V(v)(t) \leq c+w(2 c) \cdot t \int_{t}^{+\infty} \frac{1}{s^{2}} \int_{t_{0}}^{s} \tau b(\tau) d \tau d s \\
& =c+w(2 c) t\left[\frac{1}{t} \int_{t_{0}}^{t} \tau b(\tau) d \tau+\int_{t}^{+\infty} b(\tau) d \tau\right] \\
& \leq c+\left[w(2 c) \int_{t_{0}}^{+\infty} b(\tau) d \tau\right] t \leq 2 c t,
\end{aligned}
$$

leading to $V(D) \subseteq D$. We also notice that the operator $V$ is isotone, that is, $V\left(v_{1}\right) \leq V\left(v_{2}\right)$ whenever $v_{1} \leq v_{2}$.

Since $c \leq V(c)$, by applying the Knaster-Tarski fixed point theorem [3, p. 14], we deduce that the operator $V$ has a fixed point $\tilde{v}$ in $D$. This is the pointwise limit of the sequence of functions $\left(V^{n}(c)\right)_{n \geq 1}$, where $V^{1}=V$ and $V^{n+1}=V^{n} \circ V$.

Finally, we deduce that

$$
\begin{aligned}
\tilde{v}^{\prime}(t) & =[V(\tilde{v})-c]^{\prime}=\frac{\tilde{v}(t)-c}{t}-\frac{1}{t} \int_{t_{0}}^{t} b(\tau) w\left(\frac{\tilde{v}(\tau)}{\tau}\right) d \tau \\
& \leq \frac{\tilde{v}(t)-c}{t}, \quad t \geq t_{0} .
\end{aligned}
$$

The proof is complete. 
REMARK 1. It is not necessary that the solution $\tilde{v}$ of equation (5) obtained in Proposition 1 be bounded. In fact, if we replace (6) with the stronger hypothesis

$$
\int_{t_{0}}^{+\infty} t b(t) d t<+\infty
$$

then L'Hospital's rule yields

$$
\begin{aligned}
\lim _{t \rightarrow+\infty} \tilde{v}(t) & =\lim _{t \rightarrow+\infty} V(\tilde{v})(t)=c+\int_{t_{0}}^{+\infty} \tau b(\tau) w\left(\frac{\tilde{v}(\tau)}{\tau}\right) d \tau \\
& \leq c+w(2 c) \int_{t_{0}}^{+\infty} \tau b(\tau) d \tau<+\infty
\end{aligned}
$$

The ordinary differential equation

$$
v^{\prime \prime}+\frac{1}{t^{2} \sqrt{\ln t}} \sqrt{v}=0, \quad t \geq t_{0}=e^{2},
$$

has the unbounded solution $\tilde{v}(t)=\ln t$ in $\left[t_{0},+\infty\right)$ that verifies the conclusion of Proposition 1. Here, $b(t)=t^{-3 / 2}(\ln t)^{-1 / 2}, w(u)=\sqrt{u}$ and $c=1$.

REMARK 2. Proposition 1 is in perfect agreement with the conclusion of [1, Lemma 2]. The latter result, however, uses in an essential manner the fact that $w \in C^{1}$. We mention that Constantin's theorem provides an interesting complement to old contributions by Staikos and Philos; see [8].

\section{Positive solution to equation (1)}

The following result is needed in our investigation.

PROPOSITION 2 (see $[1,4]$ ). If there exist a nonnegative subsolution $v_{1}$ and a positive supersolution $v_{2}$ to equation (1) in $G_{A}$, such that $v_{1}(x) \leq v_{2}(x)$ for $x \in \bar{G}_{A}$, then (1) has a solution $u$ in $G_{A}$ such that $v_{1} \leq u \leq v_{2}$ throughout $\bar{G}_{A}$. In particular, $u=v_{2}$ on $|x|=A$.

Our main contribution here is given next.

Theorem 1. Assume that there exists $\alpha \in(0,1)$ such that $f \in C^{\alpha}(M \times J, \mathbb{R})$ for every compact set $M \subset G_{A}$ and every compact interval $J \subset \mathbb{R}$, and $g \in C^{1}([A,+\infty),[0,+\infty))$. Suppose further that (2) and (4) hold true.

Then equation (1) has a positive solution $u$, defined in $G_{B}$ for some $B>A$, such that $\lim _{|x| \rightarrow+\infty} u(x)=0$.

PROOF. Consider the positive, twice continuously differentiable functions given by

$$
U(x)=y(r)=\frac{\tilde{v}(t)}{t}, \quad t \geq t_{0},
$$


where

$$
r=|x|=\beta(t)=\left(\frac{t}{n-2}\right)^{1 /(n-2)} \quad \text { and } \quad t_{0} \geq \max \left\{1,(n-2) A^{n-2}\right\} .
$$

Here, $\tilde{v}$ is the solution of equation (5) obtained in Proposition 1 for $c=\zeta / 2$.

By a straightforward computation,

$$
t \beta^{\prime}(t)=\frac{1}{n-2} \beta(t)
$$

and

$$
\left\{\begin{array}{l}
\frac{d \tilde{v}}{d t}=y+t \beta^{\prime}(t) \frac{d y}{d r}, \\
\frac{d^{2} \tilde{v}}{d t^{2}}=\frac{n-1}{n-2} \beta^{\prime}(t) \frac{d y}{d r}+\frac{\beta(t) \beta^{\prime}(t)}{n-2} \frac{d^{2} y}{d r^{2}} .
\end{array}\right.
$$

Further, taking into account (8) and (9),

$$
\begin{aligned}
& r^{n-1}(\Delta U+f(x, U)+g(|x|) x \cdot \nabla U) \\
&= \frac{d}{d r}\left(r^{n-1} \frac{d y}{d r}\right)+r^{n-1} f(x, U)+r^{n} g(r) \frac{d y}{d r} \\
&= \frac{n-2}{\beta(t) \beta^{\prime}(t)}[\beta(t)]^{n-1}\left[\tilde{v}^{\prime \prime}(t)+\frac{1}{n-2} \beta(t) \beta^{\prime}(t) f(x, U)\right. \\
&\left.+\beta(t) \beta^{\prime}(t) g(\beta(t))\left(\tilde{v}^{\prime}(t)-\frac{\tilde{v}(t)}{t}\right)\right],
\end{aligned}
$$

for any $t \geq t_{0}$.

We have obtained that

$$
\begin{aligned}
& |x|^{n-1}(\Delta U+f(x, U)+g(|x|) x \cdot \nabla U) \\
& \quad \leq \frac{n-2}{\beta(t) \beta^{\prime}(t)}[\beta(t)]^{n-1}\left[\tilde{v}^{\prime \prime}(t)+b(t) w\left(\frac{\tilde{v}(t)}{t}\right)\right]=0,
\end{aligned}
$$

where $b(t)=\beta(t) \beta^{\prime}(t) a(\beta(t))$.

Now, $U$ is a positive super-solution of (1). Also, the trivial solution of equation (1) is its (nonnegative) sub-solution. According to Proposition 2 (see [1, 4]) there exists a nonnegative solution $u$ to (1), defined in $\bar{G}_{B}$ for $B>A$ large enough (recall (7)). Since

$$
(\Delta+g(|x|) x \cdot \nabla)(-u)=f(x, u) \geq 0,
$$

the strong maximum principle [6] can be applied to $-u$. This means that the function $-u$ cannot attain a nonnegative maximum at a point of $G_{B}$ unless it is constant. Since $-u$ is negative on $\{x:|x|=B\}$ and $-u(x) \leq 0$ throughout $\bar{G}_{B}$ as $u$ is confined between 0 and a positive super-solution $U$, it follows that $-u$ cannot have zeros.

We conclude that $u$ is a positive solution of (1) that decays to 0 when $|x| \rightarrow+\infty$. The proof is complete. 


\section{References}

[1] A. Constantin, 'Existence of positive solutions of quasilinear elliptic equations', Bull. Austral. Math. Soc. 54 (1996), 147-154.

[2] - 'Positive solutions of quasilinear elliptic equations', J. Math. Anal. Appl. 213 (1997), 334339.

[3] J. Dugundji and A. Granas, Fixed Point Theory, Vol. I (Polish Sci. Publ., Warsaw, 1982).

[4] M. Ehrnström, 'Positive solutions for second-order nonlinear differential equations', Nonlinear Anal. TMA 64 (2006), 1608-1620.

[5] M. Ehrnström and O. G. Mustafa, 'On positive solutions of a class of nonlinear elliptic equations', Nonlinear Anal. TMA 67 (2007), 1147-1154.

[6] L. E. Fraenkel, Introduction to Maximum Principles and Symmetry in Elliptic Problems (Cambridge University Press, Cambridge, 2000).

[7] D. Gilbarg and N. S. Trudinger, Elliptic Partial Differential Equations of Second Order (Springer, Berlin, 2001).

[8] V. A. Staikos and C. G. Philos, 'Non-oscillatory phenomena and damped oscillations', Nonlinear Anal. TMA 2 (1978), 197-210.

[9] Z. Yin, 'Monotone positive solutions of second-order nonlinear differential equations', Nonlinear Anal. TMA 54 (2003), 391-403.

OCTAVIAN G. MUSTAFA, Faculty of Mathematics, D.A.L.,

University of Craiova, Romania

e-mail: octaviangenghiz@yahoo.com

Corresponding address: Str. Tudor Vladimirescu, Nr. 26, 200534 Craiova, Romania 